\title{
On a Problem of A. Rotkiewicz
}

\section{By Péter Kiss and Bui Minh Phong}

\begin{abstract}
For any fixed positive integers $a, k \geqslant 2$ there are infinitely many composite integers $n$ such that $a^{n-k} \equiv 1(\bmod n)$.
\end{abstract}

1. Introduction. A. Rotkiewicz asked in his book the following question. "Let $a, k>1$ be fixed positive integers. Do there exist infinitely many composite integers $n$ such that $n \mid\left(a^{n-k}-1\right)$ ?" [5, problem 18, p. 138]. It is well known that the answer is affirmative in the case $k=1$; the numbers satisfying the condition are called pseudoprime numbers to base $a$. A general result was obtained by A. Makowski [2]: For any natural number $k \geqslant 2$ there are infinitely many composite $n$ such that

$$
a^{n-k} \equiv 1(\bmod n)
$$

for any positive integer $a$ with $(a, n)=1$. This result was proved earlier by D. C. Morrow [3] in the case $k=3$. In his proof, Makowski showed that there are infinitely many integers $n$ of the form $n=k \cdot p$ (where $p$ is a prime) such that congruence (1) holds for any positive integer $a$ if $(a, n)=1$. Naturally, $(k, a)=1$ for these numbers, and so the question remained unanswered if $a$ and $k$ are fixed and $(k, a)>1$. In the cases $(k, a)>1$, A. Rotkiewicz obtained two results: He proved that (1) has infinitely many solutions $n$ if $k=3$ and $a$ is an arbitrarily fixed positive integer, or if $k=2$ and $a=2$ (see [5, Theorem 32, p. 129] and [6], respectively).

The aim of this paper is to give a general solution of the problem. We prove:

THEOREM. Let $a(\geqslant 2)$ and $k$ be fixed positive integers. Then there are infinitely many composite integers $n$ such that

$$
a^{n-k} \equiv 1(\bmod n) .
$$

2. Auxiliary Results. We shall use some lemmas in the proof of our theorem.

LEMMA 1. Let

$$
\Phi_{n}(x)=\prod_{d \mid n}\left(x^{d}-1\right)^{\mu(n / d)}
$$

be the nth cyclotomic polynomial, where $\mu$ is the Moebius function. If $a$ and $n$ are natural numbers with $a \geqslant 2$ and $n>30$, then

$$
\Phi_{n}(a)>n(2 n+1) \text {. }
$$

Received October 25, 1985.

1980 Mathematics Subject Classification. Primary 10A10.

Key words and phrases. Congruence, primitive prime divisor. 
Proof. First we prove the inequality

$$
\Phi_{n}(a)>a^{\frac{2}{3} \varphi(n)}
$$

for every integer $a, n>1$, where $\varphi$ denotes the Euler function.

Let $\nu(n)$ denote the number of distinct prime factors of $n$. For integers $1<n \leqslant 12$ and $n=30$, using the definition of $\Phi_{n}(a)$, inequality (3) can be seen directly. For the others, separating the cases $\nu(n)=1,2,3$, and $\nu(n) \geqslant 4$, it can be easily seen that

$$
\frac{1}{3} \varphi(n) \geqslant 2^{\nu(n)-1} .
$$

But G. D. Birkhoff and H. S. Vandiver [1] showed that

$$
\Phi_{n}(a)>a^{\varphi(n)-2^{\nu(n)-1}}
$$

and so (3) indeed holds.

It is known that $\varphi(n)>n^{2 / 3}$ for $n>30$ (see, e.g., [4, p. 38]); therefore, by (3) we have

$$
\Phi_{n}(a)>a^{\frac{2}{3} n^{2 / 3}}
$$

if $n>30$. One can check that

$$
a^{\frac{2}{3} n^{2 / 3}}>n(2 n+1)
$$

for $n \geqslant 99$ if $a=2$, and for $n \geqslant 35$ if $a \geqslant 3$. In the case $a \geqslant 3$, inequality (2) can be seen directly for $n=31,32,33$ and 34 ; thus we have to prove the lemma only for $a=2$ and for integers $n$ for which $30<n<99$.

If $n>30$ and $n$ is a prime or a prime power (i.e., $\nu(n)=1$ ), then obviously

$$
\Phi_{n}(2)>2^{n / 2}>n(2 n+1) .
$$

If $\nu(n)=2$, then $\varphi(n) \geqslant n\left(1-\frac{1}{2}\right)\left(1-\frac{1}{3}\right)=n / 3$, and by (4) we have

$$
\Phi_{n}(2)>2^{n / 3-2}>n(2 n+1)
$$

for $n \geqslant 42$; by numerical calculation we can show that $\Phi_{n}(2)>n(2 n+1)$ for $30<n<42$, too.

If $\nu(n)=3$ then, similarly as above, $\varphi(n) \geqslant \frac{4}{15} n$ and

$$
\Phi_{n}(2)>2^{\frac{4}{15} n-4}>n(2 n+1)
$$

follow for $n \geqslant 64$. But there are only two integers $n=42=2 \cdot 3 \cdot 7$ and $n=60=$ $2^{2} \cdot 3 \cdot 5$ for which $\nu(n)=3$ and $30<n<64$, and by numerical computation we get $\Phi_{42}(2)>42 \cdot(2 \cdot 42+1)$ and $\Phi_{60}(2)>60 \cdot(2 \cdot 60+1)$; thus the lemma holds in this case.

If $\nu(n)>3$, then $n>99$, which completes the proof of the lemma.

LEMMA 2. Let $a(\geqslant 2)$ be a natural number and let $p(\geqslant 3)$ be a prime. If the number $a$ belongs to the exponent $(p-1) / 2$ modulo $p$ (i.e., $p \mid\left(a^{(p-1) / 2}-1\right)$ but $p+\left(a^{i}-1\right)$ for $\left.0<i<(p-1) / 2\right)$, and $P(n)$ denotes the greatest prime factor of $n$ with $P(1)=1$, then

$$
\Phi_{(p-1) / 2}(a)>p \cdot P\left(\frac{p-1}{2}\right),
$$

unless $(p ; a)=(3 ; 4),(5 ; 4),(5 ; 9),(7 ; 2),(7 ; 4),(13 ; 4),(17 ; 2)$ or $(41 ; 2)$. 
Proof. Since $P((p-1) / 2) \leqslant(p-1) / 2$ by Lemma 1 , inequality (5) holds for any $a \geqslant 2$ and $p$ if $(p-1) / 2>30$, that is, if $p>61$. For primes $p \leqslant 61$, Lemma 2 can be checked by numerical computation.

For example, in the case $p=7$ we have $\Phi_{3}(a)>3 \cdot 7$ for $a>4$, and of the numbers $a=2,3,4$ only $a=2$ and $a=4$ belong to the exponent $(p-1) / 2=3$ modulo 7. Or another example: if $p=37$, then $P=(18)=3$ and $\Phi_{18}(a)>37 \cdot 3$ for $a>2$; however, $a=2$ does not belong to the exponent 18 modulo 37 since $37+\left(2^{18}-1\right)$.

LEMMA 3. Let $a, k$, and $m$ be positive integers satisfying $a>1, m-k>1$, and $(a, m)=1$. Let a belong to the exponent $h(m)$ modulo $m$. If $h(m) \mid(m-k)$ but $h(m)<m-k$, then congruence (1) has infinitely many composite $n$-solutions, unless $m-k=2$ and $a+1$ is a power of 2 , or $m-k=6$ and $a=2$.

Proof. Let $a, k$, and $m$ be integers satisfying the conditions of the lemma. $n=m$ satisfies congruence (1) since $h(m) \mid(m-k)$. As it is well known, for any integer $n>1$ there is a prime $q$ such that $a$ belongs to the exponent $h(q)=n$ modulo $q$, unless $n=2$ and $a+1$ is a power of 2 , or $n=6$ and $a=2$ (see [1] or [7]). Thus, there exists a prime $p$ for which $h(p)=m-k$. Since $h(m)<h(p)=m-k$ and $h(m) \mid(m-k)$, we have $p+m$ and $h(m p)=m-k$. On the other hand, $h(p)=$ $m-k$ implies that $(m-k) \mid(p-1)$, and so $m p-k=(m-k) p+k(p-1)$ is divisible by $h(m p)=m-k$. From this fact it follows that $n=m p$ also satisfies congruence (1), and one can easily see that $m p-k>2$ if $a>2$ and $m p-k>6$ if $a=2$; furthermore, $h(m p)=m-k<m p-k$. Continuing this process, we get infinitely many solutions of (1).

3. Proof of the Theorem. Let $a$ and $k$ be fixed positive integers. Using the results of Makowski and Rotkiewicz mentioned above, we may assume that

$$
\begin{gathered}
(k, a)>1, \\
k \neq 3
\end{gathered}
$$

and

$$
a=2 b \geqslant 4 \text { if } k=2 \text {, }
$$

where $b$ is an integer.

First let $k=2$ and so, by (8), $a \geqslant 4$ is an integer of the form $a=2 b$. If $a=4$ and $m=7 \cdot 11=77$, then $h(7)=3$, since $7 \mid\left(4^{3}-1\right)$ but $7+\left(4^{i}-1\right)$ for $i=1,2$, and similarly $h(11)=5$. From this it follows that $h(77)=15$, and using Lemma 3 with $m=77$, we get infinitely many solutions of (1).

In the case $k=2, a=2 b>4$, Lemma 3 with $m=a-1$ also yields the proof of the Theorem, since in this case $h(m)=1$ is a divisor of $m-k$ and $h(m)<m-k$ $=a-3$.

Now let $k \geqslant 4$. As we have seen above, there is a prime $p$ such that $h(p)=k-1$, since $k-1>2$ and, by (6), $k-1 \neq 6$ if $a=2$. For this prime $p$, Fermat's congruence theorem implies that $p-k=(p-1)-(k-1)$ is divisible by $h(p)=$ $k-1$; furthermore, $p-k \neq 0$ by (6), and so obviously $p-k \geqslant h(p)=k-1 \geqslant 3$ 
and $p-k \neq 6$ if $a=2$. Thus the assertion of the Theorem follows from Lemma 3 with $m=p$ if $p-k \neq h(p)=k-1$. If $p-k=h(p)=k-1$ and $h(p)=h\left(p^{2}\right)$, then our assertion can be seen with $m=p^{2}$ similarly as above, since $p^{2}-k=$ $\left(p^{2}-1\right)-(k-1)$ is divisible by $h\left(p^{2}\right)=k-1$.

Thus, in the sequel we may assume that $k \geqslant 4$ and $p$ is a prime such that $h(p)=k-1, p-k=k-1$, and $h(p) \neq h\left(p^{2}\right)$.

Let $n \geqslant 2$ be an integer and let $\left\{p_{1}, \ldots, p_{r}\right\}$ be the set of all primitive prime divisors of $a^{n}-1$; i.e., $h\left(p_{i}\right)=n$ for $i=1, \ldots, r$. If $e_{i}>0$ is the greatest integer for which $p_{i}^{e_{i}} \mid\left(a^{n}-1\right), i=1, \ldots, r$, then

$$
\Phi_{n}(a)=\lambda \cdot \prod_{i=1}^{r} p_{i}^{e_{i}},
$$

where $\lambda=1$ or $P(n)$ (see, e.g., [1]). Since by our assumption $h(p)=k-1=$ $(p-1) / 2 \geqslant 3$ and $h(p) \neq h\left(p^{2}\right)$, Lemma 2 and (9) imply that there is a prime $q$ for which $q \neq p$ and $h(q)=(p-1) / 2=k-1$, unless $(p ; a)$ is one of the pairs of integers listed in Lemma 2. For this prime, $h(q) \mid(q-k)$ and $h(q)<q-k$, since otherwise $p=q$ would follow. Using Lemma 3 with $m=q$, the Theorem follows in this case.

If $(p ; a)$ is one of the pairs listed in Lemma $2, k \geqslant 4$ and $p-k=k-1=h(p)$, then $k=(p+1) / 2$ and so $(k ; a)=(2 ; 4),(3 ; 4),(3 ; 9),(4 ; 2),(4 ; 4),(7 ; 4),(9 ; 2)$ or $(21 ; 2)$. Since we have proved the Theorem in the case $k=2$, by $(6)$ and (7) we have to deal only with the cases $(k ; a)=(4 ; 2)$ and $(4 ; 4)$.

Using the computer TPA 11-40, we have checked that $n \mid\left(a^{n-k}-1\right)$ if $n=40369$ $=7 \cdot 73 \cdot 79$ in the case $a=2, k=4$, and if $n=19 \cdot 31=589$ in the case $a=4$, $k=4$. These numbers $n$ are composite, and so $h(n)<n-k$. By Lemma 3 , this completes the proof of the Theorem.

We note that in the cases $(k ; a)=(4 ; 2)$ and $(4 ; 4)$ the number $n=7$ satisfies congruence (1), but it does not imply infinitely many solutions since the condition $h(m)<m-k$ of Lemma 3 does not hold for $m=7$. For some pairs $(k ; a)$ we give below a table of the least composite integers $n$ which satisfy congruence (1). In some cases, (1) holds for primes less than the numbers given in the table; these cases are $(k ; a ; n)=(3 ; 4 ; 5),(4 ; 2 ; 7),(4 ; 4 ; 7),(5 ; 5 ; 13)$, and $(6 ; 2 ; 31)$.

We would like to thank G. Dorkó and A. Láng for their valuable help in the numerical computations.

\begin{tabular}{l|l|l|l|l|l}
\hline$a$ & \multicolumn{1}{c|}{2} & \multicolumn{1}{c|}{3} & \multicolumn{1}{c|}{4} & \multicolumn{1}{c|}{5} & \multicolumn{1}{c}{6} \\
\hline 2 & $20737=89 \cdot 233$ & $9=3^{2}$ & $40369=7 \cdot 73 \cdot 79$ & $25=5^{2}$ & $18631=31 \cdot 601$ \\
\hline 3 & $4=2^{2}$ & $9299=17 \cdot 547$ & $8=2^{3}$ & $25=5^{2}$ & $8=2^{3}$ \\
\hline 4 & $77=7 \cdot 11$ & $9=3^{2}$ & $589=19 \cdot 31$ & $15=3 \cdot 5$ & $9=3^{2}$ \\
\hline 5 & $4=2^{2}$ & $9=3^{2}$ & $6=2 \cdot 3$ & $62=2 \cdot 31$ & $8=2^{3}$ \\
\hline
\end{tabular}

Department of Mathematics

Teacher's Training College

Leányka u. 4

3301 Eger, Hungary

1. G. D. Birkhoff \& H. S. VAndiver, “On the integral divisors of $a^{n}-b^{n}$," Ann. of Math. (2), v. 5 , 1904, pp. 173-180.

2. A. MakowSKI, “Generalization of Morrow’s D numbers,” Simon Stevin, v. 36, 1962, p. 71. 
3. D. C. Morrow, “Some properties of D numbers," Amer. Math. Monthly, v. 58, 1951, pp. 329-330.

4. D. S. Mitrinovic \& M. S. Popadic, Inequalities in Number Theory, Naucni Podmladak, Univ. of Niš, 1978, 183 pp.

5. A. RotkiEwICZ, Pseudoprime Numbers and Their Generalizations, Student Assoc. of the Faculty of Sci., Univ. of Novi Sad, 1972, 169 pp.

6. A. Rotkiewicz, “On the congruence $2^{n-2} \equiv 1(\bmod n)$," Math. Comp., v. 43, 1984, pp. 271-272.

7. K. Zsigmondy, “Zur Theorie der Potenzreste,” Monatsh. Math., v. 3, 1892, pp. 265-284. 